\title{
Possible prophylactic anti-excitotoxic and anti-oxidant effects of virgin coconut oil on aluminium chloride-induced Alzheimer's in rat models
}

\author{
Badrah Saeed Ali Alghamdi ${ }^{\text {a,b,* }}$ \\ ${ }^{a}$ Department of Physiology, Faculty of Medicine, King Abdulaziz University, Jeddah, Saudi Arabia \\ ${ }^{\mathrm{b}}$ Neuroscience Unit, Faculty of Medicine, King Abdulaziz University, Jeddah, Saudi Arabia
}

Received 27 March 2018

Accepted 30 April 2018

\begin{abstract}
Alzheimer's disease (AD) is a progressive neurodegenerative disease that affects an estimated 5.4 million people worldwide. However, there remains no curative treatment for the condition. A $\beta$ and hyperphosphorylated tau accumulation are the main hallmarks of the disease; they interfere with glutamate uptake and mediate glutamate excitotoxicity, oxidative stress, inflammation and neurodegeneration. As virgin coconut oil (VCO) is well-known as an antioxidant and anti-inflammatory natural compound, the purpose of the present study was to assess the possible prophylactic effect of VCO on aluminium chloride $\left(\mathrm{AlCl}_{3}\right)$ - induced $\mathrm{AD}$ in rat. Alzheimer was induced by intraperitoneal injections of aluminium chloride $\left(\mathrm{AlCl}_{3}\right)$ for 45 days ( $40 \mathrm{ml} / \mathrm{kg}$ per day), and our results showed that oral administration of VCO ( $5 \mathrm{ml}$ per day for 30 days) prior to the administration of $\mathrm{AlCl}_{3}$ significantly reduced the glutamate level in both the hippocampus and prefrontal cortex compared to an VCO nonadministrated AD group. Moreover, VCO significantly increased the glutathione (GSH) level in both the hippocampus and cortex and significantly decreased the malondialdehyde (MDA) level in only the cortex of the $\mathrm{AlCl}_{3}$-induced $\mathrm{AD}$ rat model compared to an $\mathrm{AlCl}_{3}$-induced $\mathrm{AD}$ rat model with no VCO. Our findings therefore show that $\mathrm{VCO}$ preserved the ultrastructural morphology of the hippocampus and cortex of the $\mathrm{AlCl}_{3}$-induced $\mathrm{AD}$ rat model, potentially providing protection against the neurodegeneration in AD of both cortical and hippocampal neurons. In conclusion, VCO has a potential prophylactic effect for memory enhancement, anti-excitotoxicity and antioxidants in $\mathrm{AD}$ model. $\mathrm{AD}$ is the leading cause of dementia worldwide, and it has no particular effective cure. The AD incidence rate increases with age and causes neurodegeneration and memory impairment. As virgin coconut oil (VCO) is well-known as an antioxidant and anti-inflammatory natural compound, this study focused on investigating the possible prophylactic effect of VCO on AD.
\end{abstract}

Keywords: Virgin coconut oil, glutamate, MDA, GSH, Alzheimer's disease

\section{Introduction}

Alzheimer's disease (AD) is the leading cause of dementia, and its incidence increases with age [29, 43]. Short-term memory impairment is usually the first clinical manifestation of AD in patients [35].

\footnotetext{
${ }^{*}$ Corresponding author: Badrah Saeed Ali Alghamdi, King Abdulaziz University, P.O. Box: 80200, 21589, Jeddah, Saudi Arabia. Tel.: 00966567764144; E-mail: Basalghamdi@kau.edu.sa.
} 
Increases in a patient's glutamate level, oxidative stress and inflammation are also known to play a role in the pathophysiology of $\mathrm{AD}[33,44,47,55,68,74]$.

Oxidative stress is involved in the pathophysiology of $\mathrm{AD}[8,22,27,53]$. In addition, it has been reported that $\mathrm{AD}$ patients have higher levels of lipid peroxidase $[8,27]$ and a decrease in antioxidant enzyme activity [53]. This excess production of free radicals mediates neurodegeneration and the subsequent development of AD [54]. Several sources of free radical production in AD have been reported, including $\mathrm{A} \beta$ aggregation, NADPH-oxidase (Nox), mitochondrial dysfunction and inflammation [54]. Neuroinflammation, which is mediated by microglia, is also considered one of the etiopathologies of $\mathrm{AD}[31]$ as it has been found to escalate the development of $\mathrm{AD}[15,24,56,57]$. In addition, recent data have demonstrated a correlation between an activated immune system and AD [73].

Virgin coconut oil (VCO) is usually extracted from fresh coconut using a low-heat process and no chemicals to ensure that bioactive components like polyphenols, fatty acids, sterols and tocopherols are retained in the oil $[1,4,38,50,52,70]$. It has been reported that VCO has a wide range of potential protective effects as an antioxidant, anti-inflammatory, antithrombotic, antipyretic and antihypercholesterol agent $[12,34,70,71]$. Many studies have investigated different therapeutic interventions for preventing the progression of $\mathrm{AD}$, and $\mathrm{VCO}$ has been extensively studied as a natural neuroprotectant against $\mathrm{AD}$, although not without extensive controversy [17,18,21,32]. However, despite these studies, there is still a need for further information regarding the prophylactic effects of VCO supplementation before the onset of the disease.

Several types of $\mathrm{AD}$ animal models have been used to study divergent aspects of the disease. The familial type of $\mathrm{AD}$ represents less than $1 \%$ of all $\mathrm{AD}$ cases, including the dominant genetic types like the amyloid precursor protein, presenilin 1 and presenilin 2 [61]. For this reason, transgenic animals are not ideal models for studying the more common sporadic type of $\mathrm{AD}$ [39]. The administration of some chemicals, such as colchicine, $\mathrm{A} \beta$ protein and aluminum chloride $\left(\mathrm{AlCl}_{3}\right)$, provides better models for studying the sporadic type of $\mathrm{AD}$, although it requires multiple invasive administrations of the chemical compound over a long period of time [60,65]. Aluminum is abundantly found in humans' everyday environment, including drinking water, toothpaste and cooking materials [14]. It has been reported that aluminum-mediated cellular injury targets lipids, protein and DNA, which in turn induce oxidative stress, lipid peroxidation and cell injury $[11,13,67]$. Based on these considerations, in this study $\mathrm{AlCl}_{3}$ was chosen to induce a model of sporadic $\mathrm{AD}[2,26,28]$, with the overall goal of investigating the possible prophylactic effect of $\mathrm{VCO}$ in an $\mathrm{AlCl}_{3}$-induced $\mathrm{AD}$ rat model.

\section{Materials and methods}

\subsection{Animals}

This animal study was approved by the Ethical Committee of King Abdulaziz University (599-17). A total of 18 male Wistar rats around 3 months old (approximately 300 to $350 \mathrm{~g}$ ) were used in the current study. They were kept in groups of three in cages in a quiet, no-stress, 24-hour light/dark environment and were marked for identification by painting their tails. They had a free access to water and food.

\subsection{Drugs}

$\mathrm{AlCl}_{3}(\mathrm{MW}=133.34)$ was purchased from Sigma-Aldrich $(\mathrm{UK})$. The $\mathrm{AlCl}_{3}$ was dissolved in saline and injected daily intraperitoneally (IP) at a dose of $40 \mathrm{mg} / \mathrm{kg}$ per day $[36,40,41,62]$. 
Table 1

Study Groups

\begin{tabular}{llll}
\hline Group & & \multicolumn{1}{c}{30 days } & \multicolumn{1}{c}{45 days } \\
\hline Group 1 & Control & Saline orally $(5 \mathrm{ml} /$ day $)$ & Saline IP $(40 \mathrm{mg} / \mathrm{kg} / \mathrm{day})$ \\
Group 2 & $\mathrm{AD}$ & Saline orally $(5 \mathrm{ml} /$ day $)$ & $\mathrm{AlCl}_{3} \mathrm{IP}(40 \mathrm{mg} / \mathrm{kg} / \mathrm{day})$ \\
Group 3 & $\mathrm{AD}+\mathrm{VCO}$ & Virgin coconut oil orally $(5 \mathrm{ml} / \mathrm{day})$ & $\mathrm{AlCl}_{3} \mathrm{IP}(40 \mathrm{mg} / \mathrm{kg} / \mathrm{day})$ \\
\hline
\end{tabular}

The VCO was administered orally by oral gavage at a dose of $5 \mathrm{ml}$ per day [58]. The VCO was prepared following the S. Intahphuak method [34]. Fresh coconut fruit was purchased from local supermarkets. The coconut meat was shredded, soaked in water, and then pressed to produce coconut milk. The mixture sat for 48 hours, and then the oil was isolated from the milk. The VCO was filtered, aliquoted, refrigerated and then liquefied by transferring it to a water bath just before use. It was given to rats by oral gavage at a dose of $5 \mathrm{ml}$ per day.

\subsection{Study groups}

Rats were randomly distributed into 3 groups (6 rats per group): Group 1 (Control), Group 2 (AD) and Group 3 (AD+VCO). Table 1 shows the study's timeline. Group 3 rats were given VCO at a dose of $5 \mathrm{ml}$ per day by oral gavage [58] for 1 month before the $\mathrm{AlCl}_{3}$ IP injections, while Groups 1 and 2 were only given oral saline during the first month. Subsequently, $\mathrm{AlCl}_{3}$ was injected into Groups 2 and 3 IP for 45 days at a dose of $40 \mathrm{mg} / \mathrm{kg}$ per day [62]. At the end of the second month and before stopping the $\mathrm{AlCl}_{3}$ IP injections, a memory test was conducted with Groups 1 and 2 to evaluate the memory impairment in the $\mathrm{AlCl}_{3}$-induced $\mathrm{AD}$ rat models. All subjects ( $n=6$ per group) were then evaluated by memory tasks or the novel object recognition (NOR) test.

\subsection{Assessment of short-term memory by novel object recognition test}

The NOR test has been widely used for measuring memory impairment and is considered a simple memory test with no rewards [6]. In this study the test was performed in a sound-insulated environment, and objects were carefully chosen to ensure that they could be cleaned and not be easily gnawed or pushed by the rats. Two identical objects were placed within the arena, and the rats were allowed to explore both objects for 3 minutes. After 15 minutes, one of the familiar objects was replaced with a new (novel) object [3]. Animals were given 3 more minutes to explore, and their memory was evaluated regarding the frequency and duration spent in contact with both objects. After each session, the arena and objects were cleaned to avoid odour guidance.

To evaluate the exploration time spent with the familiar and novel objects, the discrimination index (DI) was calculated as $[\mathrm{DI}=(\mathrm{TN}-\mathrm{TF}) /(\mathrm{TN}+\mathrm{TF})]$, where $\mathrm{TN}$ is the exploration time for a novel object and TF is the exploration time for a familiar object. A positive DI value indicates a longer novel object exploration time, while a negative DI value indicates a longer familiar object exploration time $[3,48]$. EthoVision XT8A, a video tracking system, was used to automatically record the experiment and calculate the different parameters (Fig. 1). Some parameters were set in EthoVision XT8A to measure the total distance moved by centimetre, frequency and duration of the nose point sniffing familiar and novel objects. 


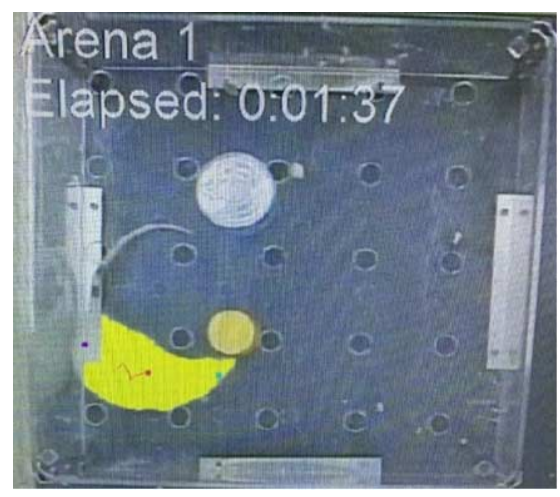

Fig. 1. EthoVision XT8A video tracking system.

\subsection{Brain tissue preparation}

After the behavioural test, the rats were euthanized by gentle decapitation following the rules and regulations of king Abdulaziz University (ethical approval no. 599-17). After dissection, their whole brains were removed carefully and washed in saline and divided in a sagittal plane into two halves. The hippocampus and prefrontal cortex of one half was immersed in $10 \%$ formalin for the histological studies or in $2 \%$ glutaraldehyde and $4 \%$ paraformaldehyde for the electron microscope studies. The hippocampus and prefrontal cortex of the other half of the brain was immediately deep-frozen in hexane and dried ice and maintained in $-80^{\circ} \mathrm{C}$ for tissue homogenate.

\subsection{Biochemical analyses}

The hippocampus and prefrontal cortexes were homogenated, and glutamate, malondialdehyde (MDA), and glutathione (GSH) levels were estimated by quantification ELIZA kits (Abcam; Cambridge, UK) following the company's recommended protocol [19].

\subsection{Histopathological analysis}

For light microscopy: thick sections, the brains were fixed in $10 \%$ formalin were subjected to gradual dehydration with assorted dilutions of alcohol. Samples were then embedded in paraffin at $56^{\circ} \mathrm{C}$ for 24 hours, sectioned by microtome ( $4 \mu \mathrm{m}$ thickness) and stained by hematoxylin and eosin.

For thin sections, immediately after sacrificing, the hippocampus and cortex were immersed in 3\% glutaraldehyde and 4\% paraformaldehyde in 0.1-M Sorensen's buffer at $\mathrm{pH} 7.4$, followed by secondary fixation in $1 \%$ osmium tetroxide, gradual dehydration, embedding and polymerization at $60^{\circ} \mathrm{C}$ for 16 hours. The samples were examined blindly by two expertise pathologists.

\subsection{Statistical analysis}

All data obtained from the study were analysed using GraphPad Prism 7.04, and all values were calculated with the mean \pm standard error. One-way ANOVA was used for comparison followed by a Tukey test and the difference was considered significant if $P<0.05$. 


\section{Results}

\subsection{VCO ameliorated short-term memory in the $\mathrm{AlCl}_{3}$-induced $A D$ rat model}

The novel object recognition test revealed a significant loss in the short-term memory of the AD rats measured by DI $(-0.12 \pm 0.17, P<0.001)$ compared to the control group $(0.83 \pm 0.13$; see Fig. 2$)$. However, VCO administration in Group 3 slightly improved short-term memory, but it did not reach a significant level $(0.27 \pm 0.14)$ compared to the AD-only group (Group 2$)$.

\subsection{VCO did not improve motor activity in the $\mathrm{AlCl}_{3}$-induced $A D$ rat model}

Measuring the total distance moved by each group revealed that $\mathrm{AlCl}_{3}$ administration $(40 \mathrm{mg} / \mathrm{kg})$ caused a significant reduction in the motor activity of the rats in the AD group (Group 2; $443.81 \mathrm{~cm} \pm$ 52.50, $n=6, P<0.0001$ ) compared to the control group (Group 1; $975.53 \mathrm{~cm} \pm 61.83, n=6$; see Fig. 2). However, VCO did not significantly improve this reduction in motor activity $(475.92 \mathrm{~cm} \pm 39.81)$ compared to the $\mathrm{AD}$ group.

\subsection{VCO improved glutamate excitotoxicity in the hippocampus and cortex of the $\mathrm{AlCl}_{3}$-induced $A D$ rat model}

The glutamate level in the AD group $\left(\mathrm{AlCl}_{3}: 40 \mathrm{mg} / \mathrm{kg}\right.$ per day) was significantly increased in the cortex $(9.99 \mathrm{nmol} / \mathrm{g} \pm 0.32 ; P<0.05)$ and hippocampus $(9.74 \mathrm{nmol} / \mathrm{g} \pm 0.35 ; P<0.01)$ compared to its control level in the cortex $(7.98 \mathrm{nmol} / \mathrm{g} \pm 0.32)$ and hippocampus $(8.27 \mathrm{nmol} / \mathrm{g} \pm 0.24$; Fig. 3). Prophylactic doses of $\mathrm{VCO}(5 \mathrm{ml}$ per day for 1 month $)$ in the AD model significantly decreased glutamate levels in both the cortex $(8.08 \mathrm{nmol} / \mathrm{g} \pm 0.31 ; P<0.05)$ and hippocampus $(8.23 \mathrm{nmol} / \mathrm{g} \pm 0.54 ; P<$ 0.05 ) compared to the AD-only group (Group 2; Fig. 3).

\subsection{VCO enhanced antioxidant activity in the hippocampus and cortex of the $\mathrm{AlCl}_{3}$-induced $A D$ rat model}

The GSH level in the AD-only group (Group 2) was significantly reduced in the cortex $(19.05 \mathrm{nmol} / \mathrm{g} \pm$ $0.59 ; P<0.05)$ and hippocampus $(17.15 \mathrm{nmol} / \mathrm{g} \pm 0.48 ; P<0.01)$ compared to the GSH level in the cortex (23.73 nmol/g \pm 2.10$)$ and hippocampus ( $23.75 \mathrm{nmol} / \mathrm{g} \pm 1.3$; Fig. 3$)$ in control group. Prophylactic doses of VCO ( $5 \mathrm{ml}$ per day for 1 month) in the AD + VCO model (Group 3) significantly increased GSH levels in both the cortex $(21.88 \mathrm{nmol} / \mathrm{g} \pm 1.37 ; P<0.05)$ and hippocampus $(20.37 \mathrm{nmol} / \mathrm{g} \pm 0.61$; $P<0.05$ ) compared to AD-only group (Fig. 3).

MDA level in the AD-only group (Group 2) was significantly increased in the adult cortex $(51.82 \mathrm{nmol} / \mathrm{g} \pm 6.16 ; P<0.0001)$ compared to the control group $(12.40 \mathrm{nmol} / \mathrm{g} \pm 1.29$; Fig. 3). However, MDA levels in the AD group did not significantly increase in the hippocampus $(13.84 \mathrm{nmol} / \mathrm{g} \pm$ 0.88 ) compared to the control group ( $12.36 \mathrm{nmol} / \mathrm{g} \pm 1.85$; Fig. 3). Prophylactic doses of VCO (5 ml per day for 1 month) in the AD + VCO group (Group 3) significantly decreased MDA levels in the cortex (16.43 nmol/g $\pm 1.46 ; P<0.0001)$ but not the hippocampus $(11.78 \mathrm{nmol} / \mathrm{g} \pm 0.73)$ compared to the AD-only group (Group 2; Fig. 3). 


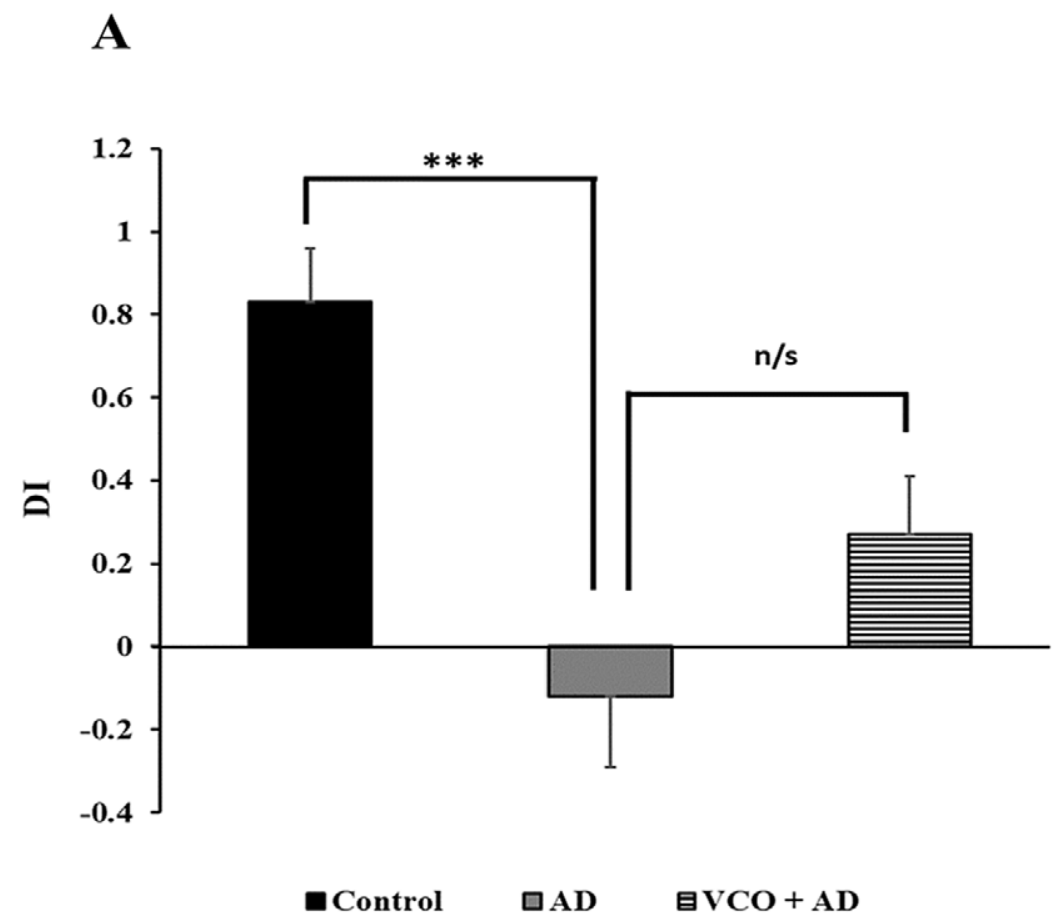

B

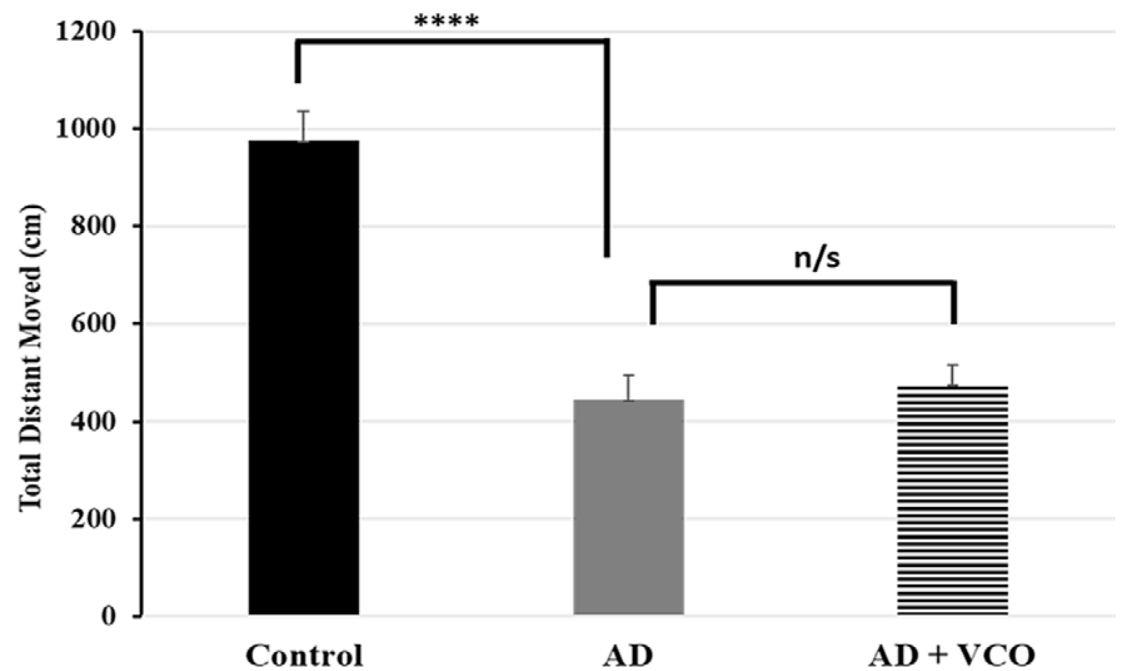

Fig. 2. (A) Discrimination index between novel and familiar objects in the different groups: A significant reduction in the DI was apparent in the AD group vs. contro), but improvement of DI in the AD + VCO group didn't reach a significant level (n/s) vs. the AD group). (B) Evaluation of the rats' motor activity by measuring the total distance moved by centimetre: A significant reduction in the total distance moved was apparent in the AD group vs. the control group but no improvement in motor activity $(\mathbf{n} / \mathbf{s})$ in the $\mathrm{AD}+\mathrm{VCO}$ group vs. the $\mathrm{AD}$. 
$\mathbf{A}$

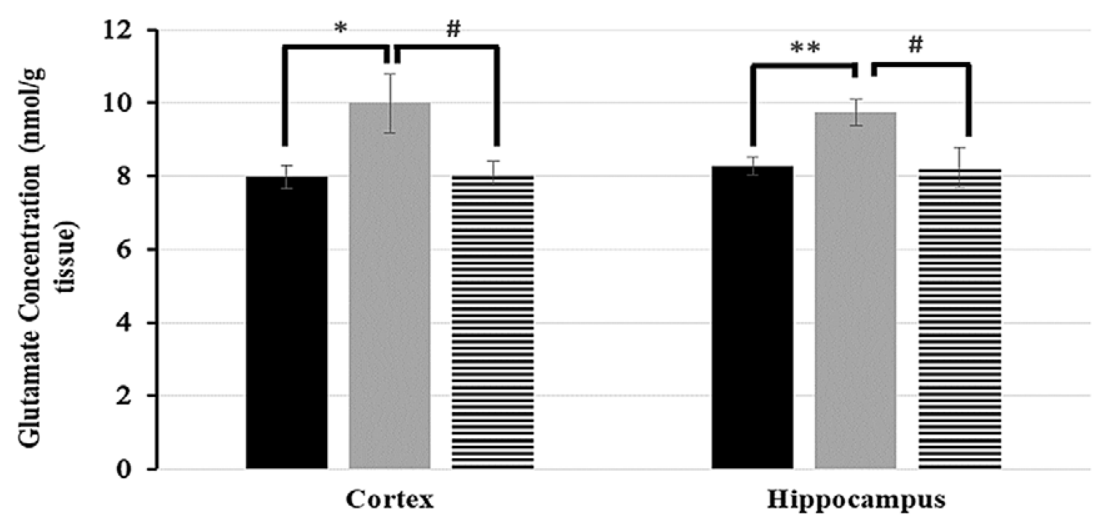

B

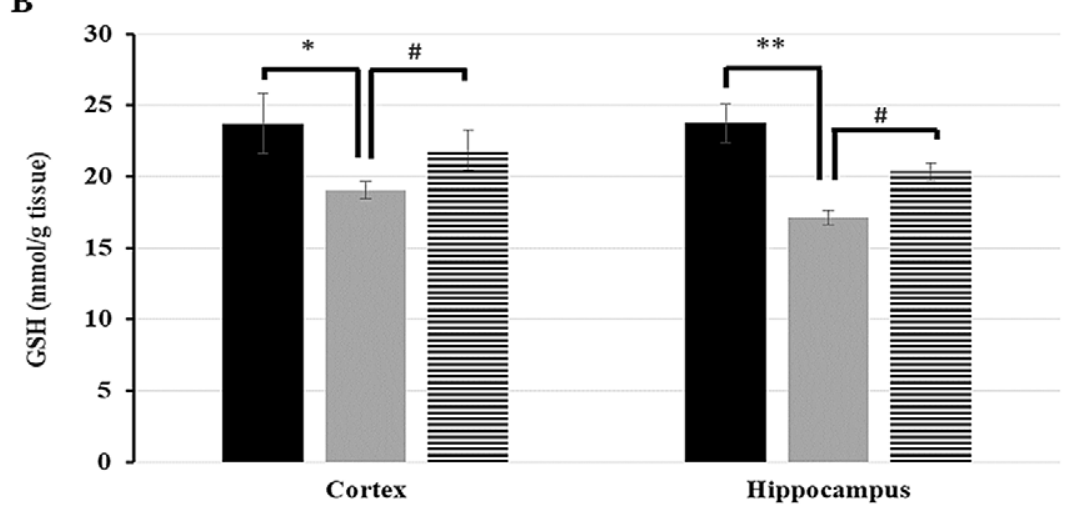

C

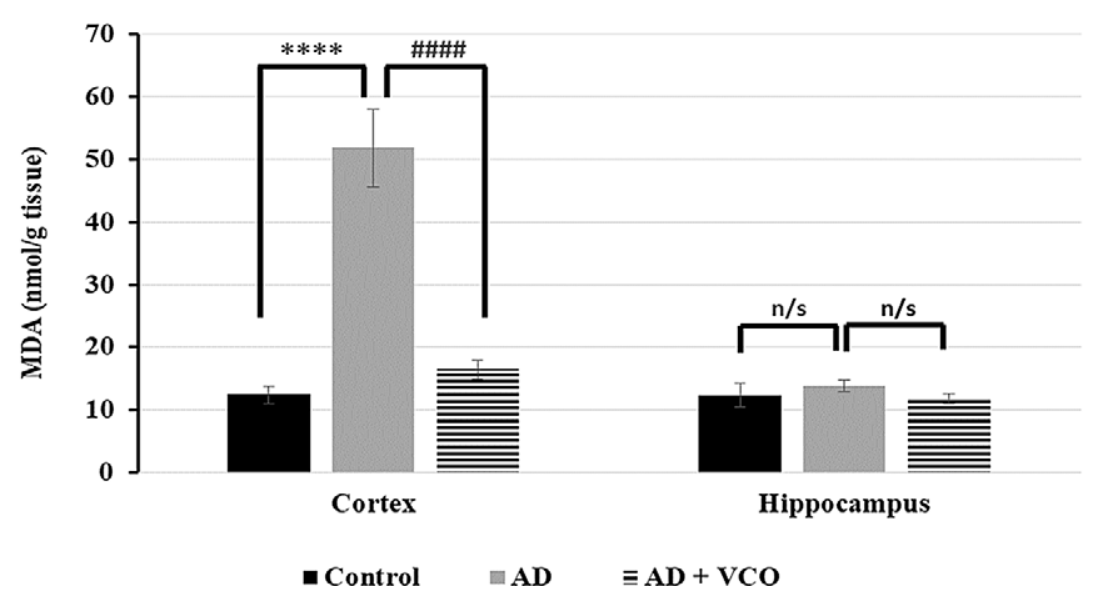

Fig. 3. Glutamate, GSH, and MDA levels in the adult rat cortex and hippocampus in different experimental groups assessed by ELIZA. (A) A significant increase in glutamate level is apparent in the AD rats vs. the control rats in the cortex and hippocampus. A significant reduction in glutamate level is shown in the AD+VCO rats vs the AD rats in the cortex and hippocampus. (B) A significant decrease in GSH level is apparent in the AD rats vs. the control rats in the cortex and hippocampus. A significant increase in GSH level is shown in the AD + VCO rats vs the AD rats in the cortex and hippocampus. (C) A significant increase in the MDA level is apparent in the $\mathrm{AD}$ rats vs. the control rats in the cortex. A significant reduction in MDA level is shown in the AD + VCO rats vs. the AD rats in the cortex. The MDA level shows nonsignificant changes in the hippocampus. 


\subsection{VCO preserved the ultrastructural morphology of the hippocampus and cortex in the $\mathrm{AlCl}_{3}$-induced $\mathrm{AD}$ rat model}

In the present study, neuronal elements of both the prefrontal cortex and hippocampal region of the control rats showed the criteria of active neurons, namely large, rounded euchromatic nuclei and lightly stained cytoplasm (Figs 4(A) and 5(A)). An electron microscope confirmed those findings and showed normal neuronal organelles, such as rough endoplasmic reticulum (rER), free ribosomes and normal mitochondria (Figs 4(B) and 5(B)).

The administration of $\mathrm{AlCl}_{3}$ resulted in degenerative changes in the $\mathrm{AD}$ groups similar to those described in the literature [37]. A large number of neurons showed irregular outlines, dark-stained cytoplasm and degenerated condensed nuclei (Figs 4(C) and 5(C)). Electron microscopy showed that the degenerated cells possessed electron-dense cytoplasm, with a dilated endoplasmic reticulum lacking ribosomes. The cytoplasm showed swollen mitochondria and numerous irregular dense bodies (Figs 4(D) and 5(D)).

The administration of VCO was found to provide potential protection against the neurodegeneration in $\mathrm{AD}$ of both cortical and hippocampal neurons, as observed by light and electron microscopy. The frequency of dark, degenerated cells was decreased, and most cells looked normal, with active euchromatic nuclei and cell organelles (Figs 4(E), 4(F), 5(E) and 5(F)).

An examination of blood capillaries from those regions showed in the AD-only group (Group 2) that there was a thickening of the basement membrane with irregularity and hypertrophy of their endothelial lining cells (Fig. 6). Those changes were not observed in the animals receiving VCO (Group 3; Fig. 6).

\section{Discussion}

The current study investigated the potential prophylactic effect of VCO before induction of AD as a natural nutrient for memory enhancement, anti-excitotoxicity and antioxidants. NOR is a simple shortterm memory test that has been widely used to assess memory impairment $[3,6]$. In the current study, there was a significant reduction in the DI in the AD model, as previously reported [2,26,49,64]. However, VCO was found to partially ameliorate memory impairment, although not at a significant level. Several explanations for this have been raised, with the most obvious being that the $\mathrm{AlCl}_{3}$ administration significantly reduced the motor activity (total distant moved) compared with the control group. This is consistent with a previous finding that showed a reduction in the activity of rats receiving $\mathrm{AlCl}_{3}$ [72]. VCO administration did not significantly improve the total distance moved by rats compared to the ADonly group, which could have affected the NOR test results. This finding agrees with previous work that proved that VCO does not improve locomotor activity [58].

$\mathrm{A} \beta$ aggregation and hyperphosphorylated tau are the hallmarks of AD pathophysiology, and they mediate oxidative stress, neuroinflammation and brain tissue injury [10,64]. It has been found that $\mathrm{AD}$ pathology interferes with glutamate uptake, which increases extracellular glutamate and mediates further excitotoxic neurodegeneration [47,55]. In the current study, the glutamate level was significantly increased in the hippocampus and cortex of the AD rats. This is consistent with previous work which revealed that glutamate excitotoxicity is an early sign of AD in which glutamanergic neurons in hippocampus and cortex are affected [9,23,25,59]. Interestingly, no previous study has investigated the effect of VCO on glutamate toxicity in the brain. In the current study, VCO was found to be a potent anti-excitotoxic, as it significantly reduced the glutamate level in the hippocampus and cortex of the $\mathrm{AD}+\mathrm{VCO}$ rats compared with the AD-only rats. 


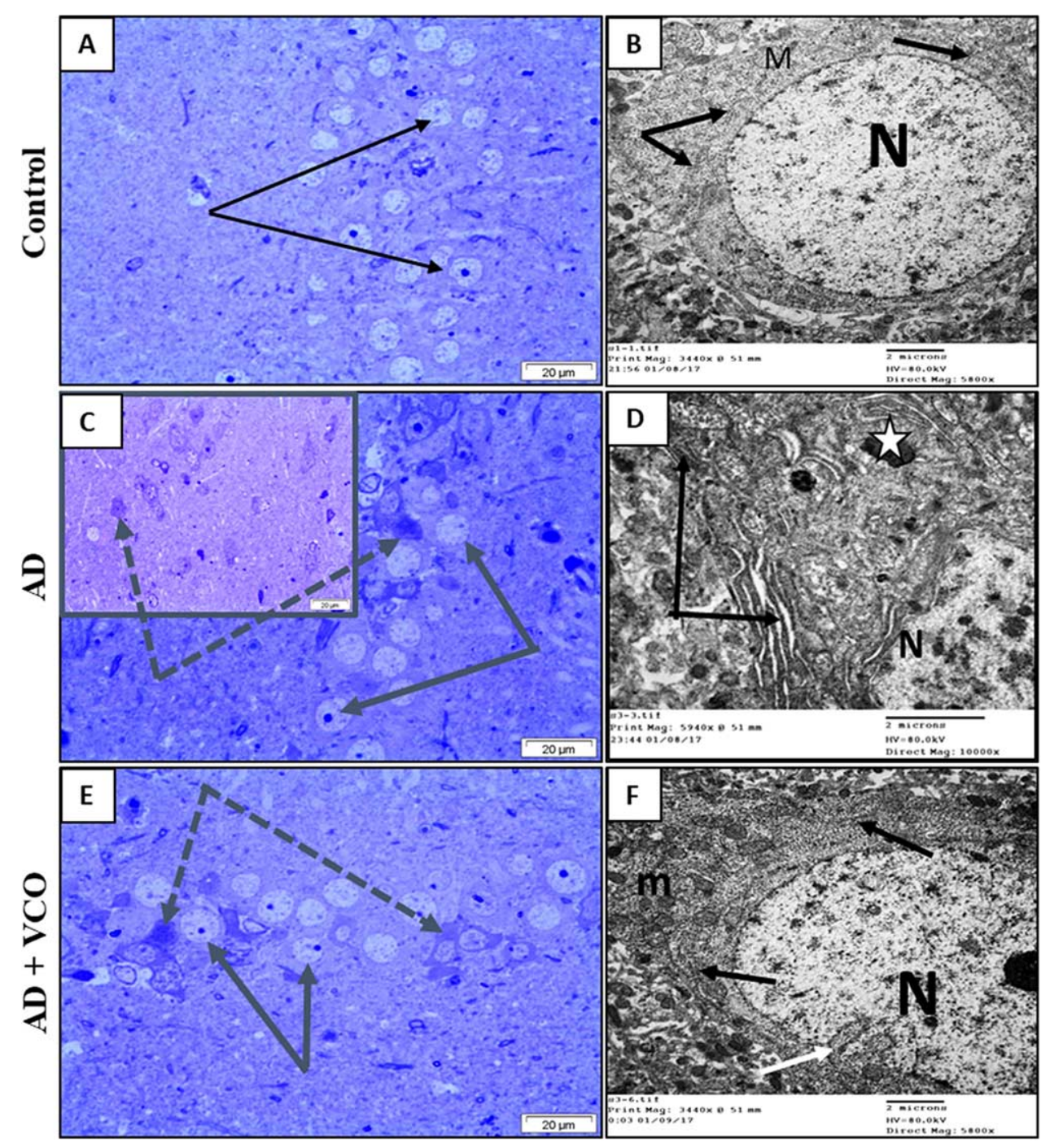

Fig. 4. Semi-thin sections and electron-ultramicrographs of rat hippocampi of the three study groups. (A) Control: The semi-thin toluidine blue-stained section shows normal neurons with vesicular nuclei and prominent nucleoli (arrows). (B) Control: electron microscope micrograph shows a normal neuron with euchromatic nucleus $(\mathrm{N})$, healthy cytoplasmic rER (black arrows) and mitochondria (M). (C) AD: Semi-thin sections show shrunken dark-stained neurons (dotted arrows). Cell nuclei are ill-defined, dark and degenerated. Few neurons looked normal with vesicular nuclei and prominent nucleoli (arrows). (D) AD: electron microscope micrograph shows a degenerated neuron with electron-dense cytoplasm and dilated rER depleted from ribosomes (black arrows). Dense bodies (lysosomes) can be seen (white star). The nucleus (N) shows clumped chromatin. (E) AD + VCO: Semi-thin sections show the preservation of normal viable neurons (black arrows). A few cells looked dark, shrunken and degenerated (dotted arrow). (F) AD + VCO: electron microscope micrograph shows a nearly normal neuron with a euchromatic nucleus $(\mathrm{N})$ and indented envelope (white arrow). The cytoplasm contains normal rER (black arrows) and mitochondria (M). Scale bar $=20 \mu \mathrm{m}$ in $\mathrm{A}, \mathrm{C}$ and $\mathrm{E}$ and $2 \mu \mathrm{m}$ in $\mathrm{B}, \mathrm{D}$ and $\mathrm{F}$.

Oxidative stress is considered one of the main causes of the neurodegenerative pathology of AD $[8,22,27,53]$. This high level of free radicals in AD can be generated from different sources, such as $\mathrm{A} \beta$ accumulation, mitochondrial dysfunction, NADPH and inflammation [54]. It has been reported that mitochondrial dysfunction, as measured by Cox activity, generates oxidative stress in the subacute phase 


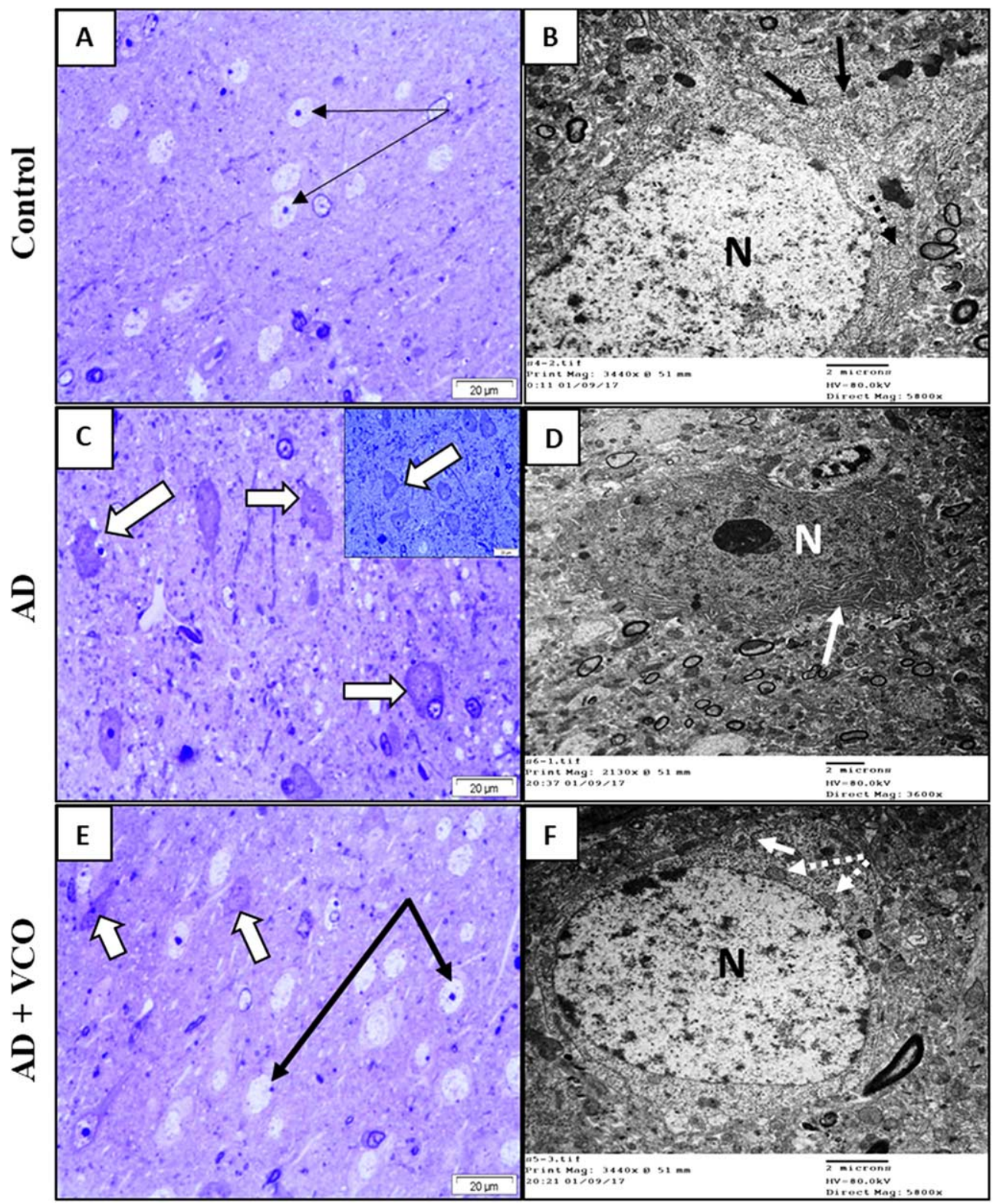

Fig. 5. Semi-thin sections and electron-ultramicrographs of rat cerebral cortexes of the three study groups. (A) Control: Semi-thin toluidine blue-stained section shows normal neurons with vesicular nuclei and prominent nucleoli (arrows). (B) Control: electron microscope micrograph shows a normal neuron with euchromatic nucleus $(\mathrm{N})$, healthy cytoplasmic rER (black arrows) and mitochondria (dotted arrow). (C) AD: Semi-thin sections show shrunken dark-stained neurons (white arrows). Cell nuclei are ill-defined, dark and degenerated. (D) AD: electron microscope micrograph shows degenerated neuron with electron dense cytoplasm and dilated rER depleted from ribosomes (white arrow). Nucleus $(\mathrm{N})$ shows clumped chromatin. (E) AD + VCO: Semi-thin sections show the preservation of active neurons (black arrows). A few cells looked dark, shrunken and degenerated (white arrows). (F) AD + VCO: electron microscope micrograph shows a nearly normal neuron with a euchromatic nucleus $(\mathrm{N})$. The cytoplasm contains normal rER (white arrows) and mitochondria (dotted white arrow). Scale bar $=20 \mu \mathrm{m}$ in $\mathrm{A}, \mathrm{C}$ and $\mathrm{E}$ and $2 \mu \mathrm{m}$ in $\mathrm{B}, \mathrm{D}$ and $\mathrm{F}$. 


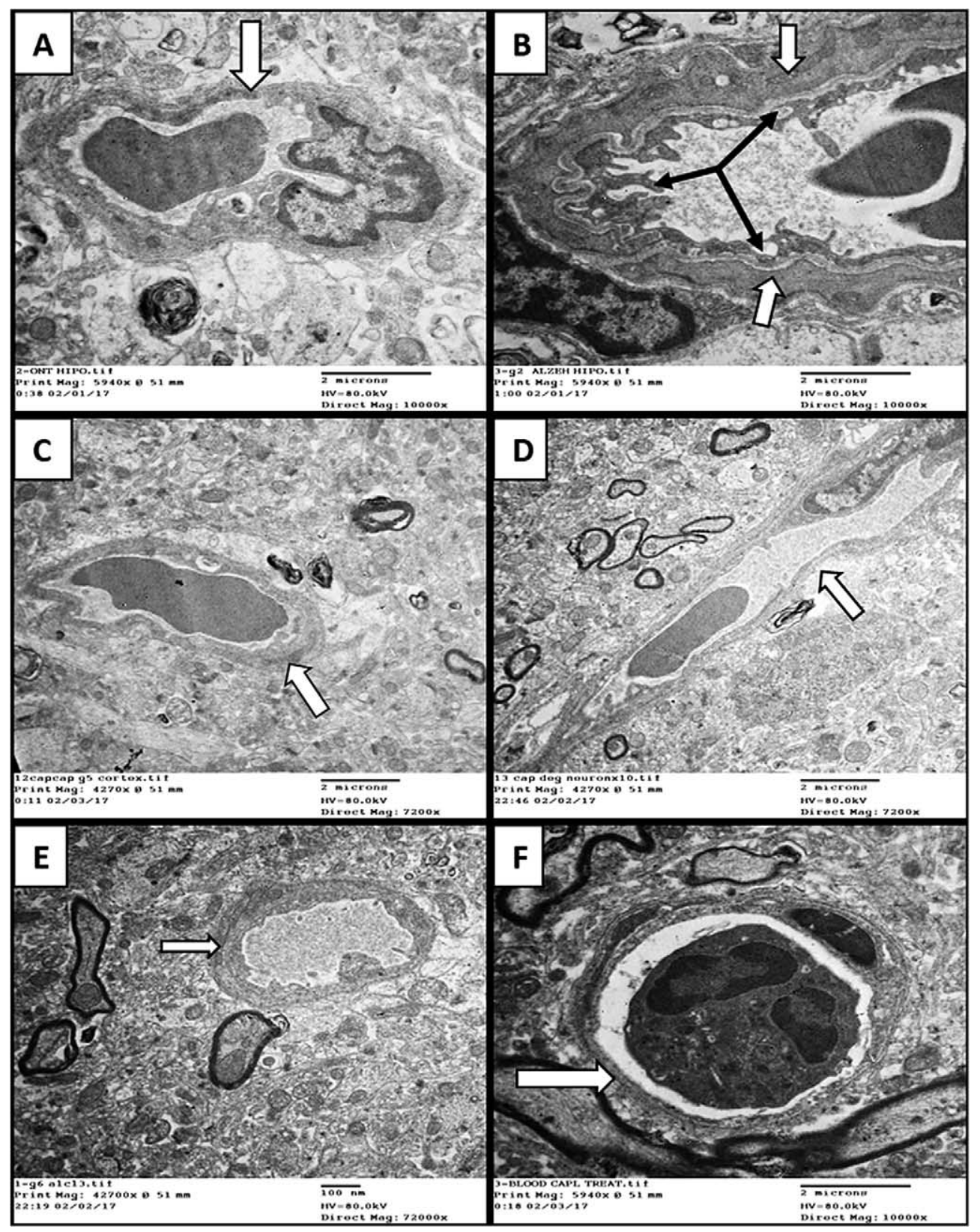

Fig. 6. Electron-ultramicrographs of blood capillaries in the three study groups. (A) Control hippocampus capillary showing normal thickness basement membrane (white arrows). (B) AD hippocampus capillary with marked thickening of basement membrane (white arrows), irregular endothelial lining and vascular degeneration (black arrows). (C) AD + VCO hippocampus capillary with normal basement membrane and endothelial lining. (D) Control cerebral cortex capillary with normal thickness basement membrane; notice the myelinated nerves nearby. (E) AD cerebral cortex capillary showing the thickening of basement membrane. (F) Cerebral cortex AD+ VCO capillary with the preservation of the normal basement membrane capillary structure (white arrow). 
of $\mathrm{AlCl}_{3}$-induced brain toxicity [66]. However, this study suggested additional sources of free radicals in the earlier acute phase of $\mathrm{AlCl}_{3}$ induction [66]. Another study reported that $\mathrm{AlCl}_{3}$ exposure promoted ROS through NADPH [46], and it has been shown that Nox is associated with the early stages of AD and that its level reflects the disease progression and severity $[5,16,63]$. Therefore, NADPH and mitochondrial dysfunction could be the main sources of free radicals in the $\mathrm{AlCl}_{3}$-induced $\mathrm{AD}$ model.

VCO is known to constitute active compounds that play an important role in antioxidation, such as polyphenol, sterol, tocopherols and squalene [1]. Moreover, it has been reported that VCO decreases the lipid peroxide level in other organs like the heart, liver and kidney [51]. This is consistent with our findings, which showed that VCO enhanced antioxidants and reduced oxidative stress in the AD rat model. However, our data also show that prophylactic doses of VCO significantly increased GSH and decreased MDA (lipid peroxidation) levels during $\mathrm{AD}$ in the cortex compared to the AD-only group. VCO also significantly increased GSH levels in the hippocampus. On the other hand, neither the ADonly group nor the $\mathrm{VCO}+\mathrm{AD}$ group saw a significant change in the MDA level in the hippocampus. This is contrary to Yang et al., who found that $\mathrm{AlCl}_{3}$ increased MDA in both the cortex and hippocampus [69]. However, a study done on the human AD brain showed an elevated level of MDA in the cortex but not in the hippocampus [7], and another study found that whole brain atrophy, not only hippocampal atrophy, distinguishes AD from mild cognitive impairment [30]. Short-term memory and, more precisely, working memory require contributions of different areas of the brain, like the temporal, parietal and prefrontal cortexes and the hippocampus $[20,42,45]$. Therefore, the impairment of one area can affect short-term memory [20]. Thus, in the current study, increasing levels of MDA in the prefrontal cortex but not the hippocampus in the AD group impaired memory.

Studying the effect of VCO at the ultrastructural level has revealed a potential protective effect of VCO in AD. VCO electron-ultramicrographs in the hippocampus and cortex showed nearly normal neurons with lighter cytoplasm staining, a euchromatic nucleus and healthy intracellular organelles like rER and mitochondria. While there were some dark, shrunken, abnormal neurons noted in the VCO + $\mathrm{AD}$ micrographs, the $\mathrm{AlCl}_{3}$-induced $\mathrm{AD}$ samples of the hippocampus and cortex showed a large number of shrunken, degenerated dark-stained neurons, with a dilated rER and clumped chromatin nuclei. The basement membrane of the blood vessels in the hippocampus and cortex appeared thickened in the AD group but normal in the VCO-treated group.

Altogether, this study provides some evidence that VCO can be used as a prophylactic in AD and that it can play a role as an anti-excitotoxic and anti-oxidant.

\section{Acknowledgements}

This work was supported by King Abdulaziz University (Grant No. 438/140/571). The author would like to extend his special thanks to Professor Soad Shaker (Anatomy Department "Histopathology", Faculty of Medicine, KAU, Jeddah, Saudi Arabia) for her generous advice and support.

\section{Conflict of interest}

The author has no conflict of interest to report. 


\section{References}

[1] M.A. Abujazia et al., The effects of virgin coconut oil on bone oxidative status in ovariectomised rat, Evid Based Complement Alternat Med 2012 (2012), 525079.

[2] M. Ahmad Rather et al., Neuroprotective role of Asiatic acid in aluminium chloride induced rat model of Alzheimer's disease, Front Biosci (Schol Ed) 10 (2018), 262-275.

[3] Z. Alrefaie and A. Alhayani, Vitamin D(3) improves decline in cognitive function and cholinergic transmission in prefrontal cortex of streptozotocin-induced diabetic rats, Behav Brain Res 287 (2015), 156-162. doi:10.1016/j.bbr.2015.03. 050.

[4] N.F. Alves et al., Coconut oil supplementation and physical exercise improves baroreflex sensitivity and oxidative stress in hypertensive rats, Appl Physiol Nutr Metab 40(4) (2015), 393-400. doi:10.1139/apnm-2014-0351.

[5] M.A. Ansari and S.W. Scheff, NADPH-oxidase activation and cognition in Alzheimer disease progression, Free Radic Biol Med 51 (2011), 171-178.

[6] M. Antunes and G. Biala, The novel object recognition memory: Neurobiology, test procedure, and its modifications, Cogn Process 13(2) (2012), 93-110.

[7] L. Balazs and M. Leon, Evidence of an oxidative challenge in the Alzheimer's brain, Neurochem Res 19(9) (1994), 11311137. doi:10.1007/BF00965146.

[8] I. Baldeiras et al., Peripheral oxidative damage in mild cognitive impairment and mild Alzheimer's disease, J Alzheimers Dis 15(1) (2008), 117-128. doi:10.3233/JAD-2008-15110.

[9] H. Braak and E. Braak, Evolution of neuronal changes in the course of Alzheimer's disease, J Neural Transm Suppl 53 (1998), 127-140.

[10] M.R. Bronzuoli et al., Targeting neuroinflammation in Alzheimer's disease, J Inflamm Res 9 (2016), 199-208. doi:10. 2147/JIR.S86958.

[11] A. Campbell, The potential role of aluminium in Alzheimer's disease, Nephrol Dial Transplant 17(Suppl. 2) (2002), 17-20. doi:10.1093/ndt/17.suppl_2.17.

[12] D.A. Cardoso et al., A coconut extra virgin oil-rich diet increases hdl cholesterol and decreases waist circumference and body mass in coronary artery disease patients, Nutr Hosp 32(5) (2015), 2144-2152.

[13] S.M. Chen et al., Hemodynamic and neuropathological analysis in rats with aluminum trichloride-induced Alzheimer's disease, PLoS One 8(12) (2013), e82561. doi:10.1371/journal.pone.0082561.

[14] M.T. Colomina and F. Peris-Sampedro, Aluminum and Alzheimer's disease, Adv Neurobiol 18 (2017), 183-197. doi:10. 1007/978-3-319-60189-2_9.

[15] C. Cunningham, Microglia and neurodegeneration: The role of systemic inflammation, Glia 61(1) (2013), 71-90. doi:10. 1002/glia.22350.

[16] S.M. de la Monte and J.R. Wands, Molecular indices of oxidative stress and mitochondrial dysfunction occur early and often progress with severity of Alzheimer's disease, J Alzheimers Dis 9(2) (2006), 167-181. doi:10.3233/JAD-2006-9209.

[17] J.E. De la Rubia Orti et al., How does coconut oil affect cognitive performance in Alzheimer patients? Nutr Hosp 34(2) (2017), 352-356.

[18] L. DeDea, Can coconut oil replace caprylidene for Alzheimer disease?, Jaapa 25(8) (2012), 19.

[19] E. Engvall and P. Perlmann, Enzyme-linked immunosorbent assay (ELISA). Quantitative assay of immunoglobulin G. Immunochemistry, 8(9) (1971), 871-874.

[20] J. Eriksson et al., Neurocognitive architecture of working memory, Neuron 88(1) (2015), 33-46. doi:10.1016/j.neuron. 2015.09.020.

[21] W.M. Fernando et al., The role of dietary coconut for the prevention and treatment of Alzheimer's disease: Potential mechanisms of action, Br J Nutr 114(1) (2015), 1-14. doi:10.1017/S0007114515001452.

[22] E. Ferreiro et al., Mitochondrial- and endoplasmic reticulum-associated oxidative stress in Alzheimer's disease: From pathogenesis to biomarkers, Int J Cell Biol 2012 (2012), 735206.

[23] P.T. Francis, Glutamatergic systems in Alzheimer's disease, Int J Geriatr Psychiatry 18(Suppl. 1) (2003), S15-S21. doi:10. $1002 /$ gps. 934.

[24] S. Gandy and F.L. Heppner, Microglia as dynamic and essential components of the amyloid hypothesis, Neuron 78(4) (2013), 575-577. doi:10.1016/j.neuron.2013.05.007.

[25] J. Gazulla and M. Cavero-Nagore, Glutamate and Alzheimer's disease, Rev Neurol 42(7) (2006), 427-432.

[26] M. Gol et al., Protective effect of raisin (currant) against spatial memory impairment and oxidative stress in Alzheimer disease model, Nutr Neurosci (2017), 1-9.

[27] J. Greilberger et al., Malondialdehyde, carbonyl proteins and albumin-disulphide as useful oxidative markers in mild cognitive impairment and Alzheimer's disease, Free Radic Res 42(7) (2008), 633-638.

[28] F. Han et al., Novel derivative of Paeonol, Paeononlsilatie sodium, alleviates behavioral damage and hippocampal dendritic injury in Alzheimer's disease concurrent with cofilin1/phosphorylated-cofilin 1 and RAC1/CDC42 alterations in rats, PLoS One 12(9) (2017), e0185102. doi:10.1371/journal.pone.0185102. 
[29] O. Hansson et al., Association between CSF biomarkers and incipient Alzheimer's disease in patients with mild cognitive impairment: A follow-up study, Lancet Neurol 5(3) (2006), 228-234. doi:10.1016/S1474-4422(06)70355-6.

[30] W.J. Henneman et al., Hippocampal atrophy rates in Alzheimer disease: Added value over whole brain volume measures, Neurology 72(11) (2009), 999-1007. doi:10.1212/01.wnl.0000344568.09360.31.

[31] F.L. Heppner, R.M. Ransohoff and B. Becher, Immune attack: The role of inflammation in Alzheimer disease, Nat Rev Neurosci 16(6) (2015), 358-372. doi:10.1038/nrn3880.

[32] I. Hu Yang et al., Coconut oil: Non-alternative drug treatment against Alzheimer's disease, Nutr Hosp 32(6) (2015), 2822-2827.

[33] M.R. Hynd, H.L. Scott and P.R. Dodd, Glutamate-mediated excitotoxicity and neurodegeneration in Alzheimer's disease, Neurochem Int 45(5) (2004), 583-595.

[34] S. Intahphuak, P. Khonsung and A. Panthong, Anti-inflammatory, analgesic, and antipyretic activities of virgin coconut oil, Pharm Biol 48(2) (2010), 151-157.

[35] H. Jahn, Memory loss in Alzheimer's disease, Dialogues Clin Neurosci 15(4) (2013), 445-454.

[36] A. Justin Thenmozhi et al., Hesperidin ameliorates cognitive dysfunction, oxidative stress and apoptosis against aluminium chloride induced rat model of Alzheimer's disease, Nutr Neurosci (2016).

[37] A. Jyoti and D. Sharma, Neuroprotective role of Bacopa monniera extract against aluminium-induced oxidative stress in the hippocampus of rat brain, Neurotoxicology 27(4) (2006), 451-457. doi:10.1016/j.neuro.2005.12.007.

[38] Y. Kamisah et al., Cardioprotective effect of virgin coconut oil in heated palm oil diet-induced hypertensive rats, Pharm Biol 53(9) (2015), 1243-1249.

[39] F.M. LaFerla and K.N. Green, Animal models of Alzheimer disease, Cold Spring Harb Perspect Med 2(11) (2012).

[40] H. Li et al., Neuroprotective effects of ginseng protein on PI3K/Akt signaling pathway in the hippocampus of Dgalactose/AlCl3 inducing rats model of Alzheimer's disease, J Ethnopharmacol 179 (2016), 162-169. doi:10.1016/j. jep.2015.12.020.

[41] W.T. Lin et al., Protective effects of low-intensity pulsed ultrasound on aluminum-induced cerebral damage in Alzheimer's disease rat model, Sci Rep 5 (2015), 9671.

[42] W.J. Ma, M. Husain and P.M. Bays, Changing concepts of working memory, Nat Neurosci 17(3) (2014), 347-356. doi:10. 1038/nn.3655.

[43] R.B. Maccioni, J.P. Munoz and L. Barbeito, The molecular bases of Alzheimer's disease and other neurodegenerative disorders, Arch Med Res 32(5) (2001), 367-381. doi:10.1016/S0188-4409(01)00316-2.

[44] K. Mahdy et al., Effect of some medicinal plant extracts on the oxidative stress status in Alzheimer's disease induced in rats, Eur Rev Med Pharmacol Sci 16(Suppl. 3) (2012), 31-42.

[45] S.G. Manohar, Y. Pertzov and M. Husain, Short-term memory for spatial, sequential and duration information, Curr Opin Behav Sci 17 (2017), 20-26. doi:10.1016/j.cobeha.2017.05.023.

[46] C.S. Martinez et al., Aluminum exposure at human dietary levels promotes vascular dysfunction and increases blood pressure in rats: A concerted action of $\mathrm{NAD}(P) \mathrm{H}$ oxidase and COX-2, Toxicology 390 (2017), 10-21.

[47] M. Matos et al., Astrocytic adenosine A2A receptors control the amyloid-beta peptide-induced decrease of glutamate uptake, J Alzheimers Dis 31(3) (2012), 555-567. doi:10.3233/JAD-2012-120469.

[48] S.A. Moosavirad et al., Protective effect of vitamin C, vitamin B12 and omega-3 on lead-induced memory impairment in rat, Res Pharm Sci 11(5) (2016), 390-396. doi:10.4103/1735-5362.192490.

[49] M. Nampoothiri et al., Insulin combined with glucose improves spatial learning and memory in aluminum chloride-induced dementia in rats, J Environ Pathol Toxicol Oncol 36(2) (2017), 159-169. doi:10.1615/ JEnvironPatholToxicolOncol.2017020185.

[50] K.G. Nevin and T. Rajamohan, Beneficial effects of virgin coconut oil on lipid parameters and in vitro LDL oxidation, Clin Biochem 37(9) (2004), 830-835. doi:10.1016/j.clinbiochem.2004.04.010.

[51] K.G. Nevin and T. Rajamohan, Virgin coconut oil supplemented diet increases the antioxidant status in rats, Food Chemistry 99(2) (2006), 260-266. doi:10.1016/j.foodchem.2005.06.056.

[52] B.S. Nurul-Iman et al., Virgin coconut oil prevents blood pressure elevation and improves endothelial functions in rats fed with repeatedly heated palm oil, Evid Based Complement Alternat Med 2013 (2013), 629329.

[53] M. Padurariu et al., Changes of some oxidative stress markers in the serum of patients with mild cognitive impairment and Alzheimer's disease, Neurosci Lett 469(1) (2010), 6-10. doi:10.1016/j.neulet.2009.11.033.

[54] M. Padurariu et al., The oxidative stress hypothesis in Alzheimer's disease, Psychiatr Danub 25(4) (2013), 401-409.

[55] K. Parameshwaran, M. Dhanasekaran and V. Suppiramaniam, Amyloid beta peptides and glutamatergic synaptic dysregulation, Exp Neurol 210(1) (2008), 7-13. doi:10.1016/j.expneurol.2007.10.008.

[56] V.H. Perry and C. Holmes, Microglial priming in neurodegenerative disease, Nat Rev Neurol 10(4) (2014), $217-224$. doi:10.1038/nrneurol.2014.38.

[57] S. Prokop, K.R. Miller and F.L. Heppner, Microglia actions in Alzheimer's disease, Acta Neuropathol 126(4) (2013), 461-477. 
[58] N.S. Rahim et al., Enhanced memory in wistar rats by virgin coconut oil is associated with increased antioxidative, cholinergic activities and reduced oxidative stress, Pharm Biol 55(1) (2017), 825-832.

[59] T.J. Revett et al., Glutamate system, amyloid ss peptides and tau protein: Functional interrelationships and relevance to Alzheimer disease pathology, J Psychiatry Neurosci 38(1) (2013), 6-23.

[60] K. Saba et al., Energetics of excitatory and inhibitory neurotransmission in aluminum chloride model of Alzheimer's disease: Reversal of behavioral and metabolic deficits by Rasa Sindoor, Front Mol Neurosci 10 (2017), 323. doi:10.3389/ fnmol.2017.00323.

[61] J.J. Sabbagh, J.W. Kinney and J.L. Cummings, Animal systems in the development of treatments for Alzheimer's disease: Challenges, methods, and implications, Neurobiol Aging 34(1) (2013), 169-183. doi:10.1016/j.neurobiolaging.2012.02. 027.

[62] A.A. Shati, F.G. Elsaid and E.E. Hafez, Biochemical and molecular aspects of aluminium chloride-induced neurotoxicity in mice and the protective role of Crocus sativus L. extraction and honey syrup, Neuroscience 175 (2011), 66-74.

[63] S. Sorce and K.H. Krause, NOX enzymes in the central nervous system: From signaling to disease, Antioxid Redox Signal 11(10) (2009), 2481-2504. doi:10.1089/ars.2009.2578.

[64] H. Syed et al., Cyclooxygenase I and II inhibitors distinctly enhance hippocampal- and cortex-dependent cognitive functions in mice, Mol Med Rep 12(5) (2015), 7649-7656. doi:10.3892/mmr.2015.4351.

[65] D. Van Dam and P.P. De Deyn, Drug discovery in dementia: The role of rodent models, Nat Rev Drug Discov 5(11) (2006), 956-970.

[66] S. Vucetic-Arsic et al., Oxidative stress precedes mitochondrial dysfunction in gerbil brain after aluminum ingestion, Environ Toxicol Pharmacol 36(3) (2013), 1242-1252. doi:10.1016/j.etap.2013.10.008.

[67] J.R. Walton, An aluminum-based rat model for Alzheimer's disease exhibits oxidative damage, inhibition of PP2A activity, hyperphosphorylated tau, and granulovacuolar degeneration, J Inorg Biochem 101(9) (2007), 1275-1284. doi:10.1016/j. jinorgbio.2007.06.001.

[68] R. Wang and P.H. Reddy, Role of glutamate and NMDA receptors in Alzheimer's disease, J Alzheimers Dis 57(4) (2017), 1041-1048. doi:10.3233/JAD-160763.

[69] W.N. Yang et al., The effects of perindopril on cognitive impairment induced by d-galactose and aluminum trichloride via inhibition of acetylcholinesterase activity and oxidative stress, Pharmacol Biochem Behav 114-115 (2013), 31-36.

[70] S.K. Yeap et al., Antistress and antioxidant effects of virgin coconut oil in vivo, Exp Ther Med 9(1) (2015), 39-42. doi:10. 3892/etm.2014.2045.

[71] Z.A. Zakaria et al., In vivo antinociceptive and anti-inflammatory activities of dried and fermented processed virgin coconut oil, Med Princ Pract 20(3) (2011), 231-236.

[72] G.F. Zeng et al., The role of 6-gingerol on inhibiting amyloid beta protein-induced apoptosis in PC12 cells, Rejuvenation Res 18(5) (2015), 413-421. doi:10.1089/rej.2014.1657.

[73] B. Zhang et al., Integrated systems approach identifies genetic nodes and networks in late-onset Alzheimer's disease, Cell 153(3) (2013), 707-720.

[74] X. Zhu et al., Mitochondrial abnormalities and oxidative imbalance in Alzheimer disease, J Alzheimers Dis 9(2) (2006), 147-153. doi:10.3233/JAD-2006-9207. 